\title{
CUESTIONES MÉDICO-LEGALES SOBRE LOS FÁRMACOS ACTUALMENTE EMPLEADOS EN EL TRATAMIENTO DE LA DMAE
}

\section{MEDICAL AND LEGAL ISSUES RELATED TO THE DRUGS CURRENTLY USED IN THE TREATMENT OF AGE-RELATED MACULAR DEGENERATION (ARMD)}

\author{
MENÉNDEZ DE LUCAS JA ${ }^{1}$, MORCILLO LAIZ R ${ }^{2}$
}

La legislación básica sobre medicamentos en nuestro País es la Ley del Medicamento (1), en la que se regula, dentro del marco de las competencias del Estado, todos los aspectos relacionados con la fabricación, elaboración, control de calidad, precios, información, publicidad, importación, exportación, prescripción, autorizaciones, registros y otras actividades o competencias que tenga algo que ver con los medicamentos. Asimismo se regula la actuación de las personas que intervengan en su comercialización o que por su titulación profesional puedan garantizarlos, controlarlos, recetarlos o dispensarlos (art. $1^{\circ}{ }^{\circ}$ ). Se prohíbe la venta de estos productos fuera de las oficinas de farmacia, así como la elaboración, fabricación, importación, exportación, distribución, comercialización, prescripción y dispensación de productos o preparados que se presentasen como medicamentos y no estuvieran legalmente reconocidos, o los remedios secretos (art. 6. $\left.{ }^{\circ}\right)$.

Tienen especial interés en relación a los fármacos actualmente empleados en el tratamiento de la DMAE, los requisitos que establece que deben seguirse para el empleo de medicamentos extranjeros que estén legalmente comercializados en algún país extranjero y no autorizados en España (art. 37). En estos casos debe rellenarse una serie de documentos (Prescripción médica en la receta correspondiente e informe del oftalmólogo recogiendo los tratamientos previos y el carácter imprescindible de la prescripción, o en su defecto, los impresos de solicitud de autorización al Ministerio: A2 y A3) porque es preceptivo que el Ministerio de Sanidad y Consumo autorice la importación en cada caso particular, para lo que se exige que resulte imprescindible para el tratamiento o diagnóstico de patologías concretas.

Además de esta ley básica sobre el manejo de fármacos, consideramos imprescindible hacer una breve referencia al Real Decreto 223/2004 de 6 de febrero, por el que se regulan los Ensayos Clínicos con Medicamentos (2), ya que en este texto legal, además de regular este tipo de investigaciones científicas, atribuye a la Agencia Española del Medicamento y Productos Sanitarios, la competencia para autorizar el uso de medicamentos sin autorización para ser comercializados, en casos individuales por razones terapéuticas, ya que hay ocasiones en las que un paciente puede necesitar un medicamento fuera de las situaciones de uso general. Este tipo de administración de medicamentos está contemplada en el artículo 28 de este texto legal y se denomina uso compasivo de medicamentos, entendiendo como tal la utilización de éstos en pacientes aislados y al margen de un ensayo clínico de medicamentos en investigación, o también la prescripción de especialidades farmacéuticas para indicaciones o condiciones de uso distintas de las autorizadas, cuando el médico, bajo su exclusiva responsabilidad considera indispensable su utilización. Este tipo de uso de medicamentos exige también la cumplimentación de una documentación (Informe del

\footnotetext{
1 Doctor en Medicina. Jefe de Servicio de Oftalmología de la Clínica Médico Forense de Madrid.

2 Doctor en Medicina. FEA del Hospital Ramón y Cajal (Madrid). Instituto de Ciencias Visuales (Madrid).

E-mail: incivi@incivi.com
} 
oftalmólogo en el que se recojan las terapias previas y la ausencia de alternativas terapéuticas, conformidad del director de hospital, documento de CI y autorización de la Agencia del Medicamento), lo pueden solicitar médicos especialistas del ámbito hospitalario a través de los Servicios de Farmacia de los Hospitales, para pacientes en régimen interno o ambulatorio. El uso compasivo de medicamentos es la herramienta legal que debe emplear el especialista para aquellos pacientes que no están incluidos en ensayos clínicos y en los que considere necesario aplicar un tratamiento no autorizado, ya que le ampararía legalmente en caso de que ocurriera alguna complicación o efecto secundario y el paciente o sus familiares decidieran denunciarle por malapraxis.

Finalmente, podría emplearse un medicamento no autorizado, pero que está en fase de investigación clínica dentro del marco de un ensayo clínico. Deja muy claro este Real Decreto, en su primer artículo, que «no tendrá la consideración de ensayo clínico la administración de un medicamento en investigación a un solo paciente en el ámbito de la práctica médica habitual con el único propósito de conseguir un beneficio terapéutico para el paciente, que se regirá por lo dispuesto sobre uso compasivo», añadiendo que «la práctica médica y la libertad profesional de prescripción del médico no ampararán, en ningún caso, la realización de ensayos clínicos no autorizados, ni la utilización de remedios secretos o no declarados a la autoridad sanitaria».

Solo hay, por lo tanto cuatro formas de administrar un medicamento a un paciente: $1 .{ }^{\mathrm{a}}$ Como medicamento autorizado (especialidades farmacéuticas registradas), 2. ${ }^{a}$ Como medicamento en fase de investigación clínica (calificado como tal por la Administración, y limitado al marco de un ensayo clínico concreto y autorizado). 3. ${ }^{a}$ Como medicamento extranjero (medicamentos legalmente comercializados en otros países, imprescindibles y autorizados por el Ministerio). 4. ${ }^{a}$ Como medicamento de uso compasivo cuando lo indique un especialista hospitalario, a un paciente en el que no exis$\tan$ otras alternativas terapéuticas o ya se hayan agotado y se considere indispensable su utilización.

Aplicando lo que hemos dicho anteriormente en relación a la legislación sobre la materia, a los medicamentos más habituales empleados actualmente en el tratamiento de la DMAE húmeda, podríamos analizar cuatro situaciones:
1. ${ }^{a}$ La terapia fotodinámica (TFD): El VISUDY$\mathrm{NE}^{\circledR}$, se emplea para tratar lesiones pequeñas, de cualquier tipo angiográfico y las grandes cuando sean predominantemente clásicas u ocultas con progresión reciente y AV $\leq 0,4$ (3); aunque las indicaciones para las que ha sido aprobado su uso son MNV (=membranas neovasculares) subfoveolares clásicas o con evidencia de progresión de la enfermedad reciente o en curso y secundarias a miopía patológica. En el caso de que nos planteáramos aplicar terapia fotodinámica a un paciente en el que no se cumplieran estas condiciones, podríamos hacerlo, pero utilizando la vía del uso compasivo ya que vamos a emplear una especialidad farmacéutica para una indicación o condición de uso distinta de la autorizada. Por lo tanto deberíamos cumplimentar una serie de documentos, en los que se haga constar que no existe otro tratamiento alternativo, o que ya se ha empleado sin conseguir buenos resultados, y que bajo la responsabilidad del especialista hospitalario que lo solicita, la TFD se considera indispensable en ese paciente. Además habrá que esperar a que la Agencia Española del Medicamento nos autorice su aplicación.

2. ${ }^{\mathrm{a}}$ La del pegaptanib $\left(M A C U G E N^{\circledR}\right)$ : En el momento actual no está autorizado su comercialización en España, aunque posiblemente lo esté dentro de poco tiempo. Al ser un medicamento cuyo uso está autorizado en otros países (lo aprobó la FDA en diciembre de 2004 y hace pocos días en Alemania), podríamos emplearlo como medicamento extranjero, si se considera imprescindible, no hay alternativa terapéutica o se ha agotado (podría ocurrir en pacientes con MNV que estuvieran fuera de las indicaciones de la TFD) o que ya se hubiera utilizado sin éxito, y lo autorice el Ministerio.

3. ${ }^{a}$ El empleo del acetónido de triamcinolona $\left(T R I G O N^{\circledR}\right)$ por vía intraocular recambiando el excipiente por BSS, debería hacerse mediante la vía del uso compasivo de medicamentos (4) ya que empleamos una especialidad farmacéutica al margen de las indicaciones para las que ha sido aprobada. Actualmente al estar disponible este medicamento autorizado para administración intraocular en EEUU, podemos aplicarlo como medicamento extranjero, siempre y cuando lo hagamos dentro de las indicaciones para las que ha sido autorizado y tengamos la precaución de que el paciente nos firme un documento de CI, al ser la inyección intraocular de medicamentos una actuación de riesgo. 
4. ${ }^{\text {a }}$ Más complicado resulta el momento actual el uso del bevacizumab (AVASTIN $\left.{ }^{\circledR}\right)$, ranibizumab $\left(\right.$ LUCENTIS ${ }^{\circledR}$ ), acetato de anecortave (REETA$N E^{\circledR}$ ), etc. ya que no podrían utilizarse por la vía del medicamento extranjero, al no estar autorizado su uso actualmente en ningún país del mundo para aplicación intraocular en el tratamiento de membranas neovasculares y tampoco podrían utilizarse mediante la vía del uso compasivo ya que existen otros tratamientos alternativos disponibles: la terapia fotodinámica o el macugen ${ }^{\circledR}$ (como medición extranjera). Solamente en el caso, muy excepcional, de que se hubieran agotado estos tratamientos sin resultados, podría plantearse su empleo compasivo. Por lo tanto, y en momento actual, el uso de estos medicamentos, está restringido, exclusivamente a los pacientes incluidos en ensayos clínicos autorizados.

De todos es conocido que los retinólogos tienen un importante riesgo de sufrir denuncias por parte de sus pacientes. Ya demostramos que esta subespecialidad es una de las que más riesgo de denuncias tiene dentro de la oftalmología (5), y vimos que hay autores que atribuyen este hecho a que la mayoría de la patología que manejan los retinólogos tiene un alto potencial de producir pérdida visual severa o incluso ceguera ya que consideran este tipo de cirugía más compleja que otras intervenciones oftalmológicas (6). Pero precisamente por eso, no tiene demasiado sentido que asuman un riesgo adicional al que necesariamente implica su actividad profesional habitual. No nos estamos refiriendo a conductas claramente ilícitas, que están fuera del ámbito de actuación de cualquier médico, como adquirir medicamentos a través de Internet, o introducirlos de contrabando en el país desde algún principado cercano. Nos referimos a aquellos compañeros oftalmólogos que, con toda su buena voluntad, siempre en beneficio del paciente y motivados por artículos científicos recientemente publicados en revistas o por comunicaciones presentadas en los congresos de la especialidad aplican a sus pacientes tratamientos con medicamentos que se encuentran en fase de investigación, fuera del contexto de un ensayo clínico autorizado.

En casi todos los congresos de Retina y en publicaciones de autores que han analizado en profundidad el tema (4), se plantea el problema del riesgo de una denuncia por mala praxis, que tienen la mayoría de los retinólogos que aplican el TRIGÓN ${ }^{\circledR}$, por una vía para la que no estaba autorizado, además de los inconvenientes que conlleva hacer el recambio del excipiente. Afortunadamente, ya lo tenemos disponible como medicamento extranjero para este uso, y suponemos que en poco tiempo se comercializará en nuestro país, aportando un cierto sosiego y paz a la actividad profesional diaria de muchos compañeros que lo utilizan habitualmente.

Es posible que en poco tiempo, se publiquen los resultados de los ensayos clínicos que se están realizando con muchos de los medicamentos antiangiogénicos de los que hemos hablado y que por el momento no está autorizado su uso. La abrumadora diferencia de precio entre algunos de ellos (7), evidentemente va a suponer un importante aliciente para la investigación, además de que posiblemente obligue a ajustar los precios, con lo que un mayor número de pacientes podrá beneficiarse de este tipo de tratamientos. Pero estas consideraciones de carácter económico, que pueden tener mucha importancia para el gerente del hospital, no deben condicionar las indicaciones que haga el médico. Probablemente serían insostenibles ante un juez, si tenemos la desgracia de que el paciente nos denuncie por haber sufrido una complicación al tratarle con un medicamento no autorizado, fuera de los supuestos legalmente previstos.

\section{NOTA}

Con posterioridad a la fecha de recepción de esta editorial, concretamente el 30 de junio de 2006, la FDA (Food and Drug Administration) de los Estados Unidos aprobó el ranibizumab (Lucentis ${ }^{\circledR}$ ) para el tratamiento de la DMAE húmeda.

\section{BIBLIOGRAFÍA}

1. Ilustre Colegio Oficial de Médicos de Madrid. Anuario Colegial 2000. 2000; vol. II capt. $2^{\circ}$ : 1153-1182.

2. Real Decreto 223/2004 de 6 de febrero, por el que se regulan los ensayos clínicos con medicamentos. BOE $n .^{\circ} 33$ de 27 de febrero de 2004; 325.

3. Bueno Lozano J, Rodríguez Ramírez M, García Lozano I, Zato MA. Nuevos tratamientos para la degeneración macular asociada a la edad. St Ophthalmol 2005; 4: 211 216.

4. Araiz Iribarren J. Triamcinolona intravítrea. Arch Soc Esp Oftalmol 2004; 79: 583-585.

5. Menéndez JA. El informe pericial oftalmológico en las reclamaciones por mala práxis. Madrid: Universidad Complutense de Madrid. 2005. 292 pp. Thesis Doctoralis. 
6. Kraushar MF. Medical malpractice experiences of vitreoretinal sepecialist: risk prevencion strategies. Retina 2003; 23: 523-529.

7. Michels S, Rosenfeld PJ, Puliafito CA, Marcus EN, Venkatraman AS. Systemic bevacizumab (Avastin) the- rapy for neovascular age-related macular degeneration twelve-weeks results of an uncontrolled openlabel clinical study. Ophthalmology 2005; 112: 1035 1047. 